\title{
Endovascular Treatment of Internal Carotid and Vertebral Artery Aneurysms Using Covered Stents
}

Ivan Vulev and Andrej Klepanec

Additional information is available at the end of the chapter

http://dx.doi.org/10.5772/48733

\section{Introduction}

\subsection{Epidemiology}

Internal carotid artery (ICA) and vertebral artery (VA) aneurysms are most frequent aneurysmatic lesions. Especially intracranial aneurysms are pathologic focal dilatations of the cerebrovasculature that are prone to rupture. These vascular abnormalities are classified by presumed pathogenesis. Saccular, berry, or congenital aneurysms constitute $90 \%$ of all cerebral aneurysms and are located at the major branch points of large arteries. Dolichoectatic, fusiform, or arteriosclerotic aneurysms account for $7 \%$ of all cerebral aneurysms. Infectious or mycotic aneurysms are situated peripherally and comprise $0.5 \%$ of all cerebral aneurysms. Other peripheral lesions include neoplastic aneurysms, rare sequelae of embolized tumor fragments, and traumatic aneurysms. Saccular intracranial aneurysms are situated in the anterior circulation in $85-95 \%$ of cases, whereas dolichoectatic aneurysms predominantly the vertebrobasilar system. Multiple saccular aneurysms are noted in $20-30 \%$ of patients with cerebral aneurysms. Aneurysmal rupture can result most often in subarachnoid hemorrhage, but may also present as intraparenchymal, intraventricular, or subdural hemorrhage. Giant saccular aneurysms, defined as greater than $25 \mathrm{~mm}$ in diameter, may cause $\mathrm{SAH}$, but these lesions more frequently produce mass effect and may result in distal thromboembolism.

On the other hand, extracranial internal carotid and vertebral artery aneurysms usually may present with cerebral embolism, transient ischemic attack, cerebrovascular insufficiency, continued enlargement with compression syndrome, vessel occlusion or hemorrhage. Aneurysms may be also often asymptomatic until the time of rupture. In the past, most of these aneurysms were treated surgically. Surgery, however, is often difficult because of the 
location and the damaged arterial wall and may result in sacrifice of the internal carotid or vertebral artery. Vertebral artery (VA) aneurysms constitute 0.5 to $3 \%$ of intracranial aneurysms and $20 \%$ of posterior circulation aneurysms [1]. The causes of aneurysms are multiple and may occur following trauma, mycotic infection, as a result of atherosclerosis, tumor invasion or radiation necrosis or iatrogenic. Among these, dysplastic lesions appeared to be the main cause of extracranial internal carotid artery aneurysms, associated or not with spontaneous dissection. VA aneurysms include VA-PICA (posterior inferior cerebellar artery) aneurysms, vertebro-basilar junction aneurysms, distal PICA aneurysms and those aneurysms located along the distal VA. Dissecting aneurysms of the intradural vertebral arteries often present with subarachnoid haemorrhage. A second episode of bleeding (rebleeding) is common and deadly. Rebleeding rates were estimated at $71 \%$ of cases with subsequent re-rupture of the aneurysms in 57\% [2]. Ruptured posterior circulation aneurysms are technically difficult to expose and clip and their management and surgical outcomes are poorer as compared to anterior circulation aneurysms [3]. They often need expertise with various skull base approaches to improve the exposure, to minimize brain retraction and to achieve better outcome. Certain subset of posterior circulation aneurysms are considered at even higher risk for surgery due to their location and the size, prompting recourse to other modalities of therapy.

\subsection{Diagnostic imaging}

Early diagnosis and evaluation of anatomical characteristics of the internal carotid and vertebral artery aneurysms are essential in terms of surgical or endovascular treatment planning.

Ultrasound imaging is initially useful in the diagnostic process for cases with suspicion for extracranial internal carotid artery aneurysm palpable pulsatile mass at neck region. It serves for determination of the size and extension of the aneurysm. Although ultrasound is a valuable diagnostic tool for definition of anechoic structure and pulsation of the aneurysm, it may become insufficient in defining thrombosed aneurysm, relation of the aneurysm with its neighboring structures. Duplex ultrasound scanning is the most simple investigation in the detection of vertebral and extracranial internal carotid artery aneurysms, but this may fail if the lesion is located high, especially if the patient has a short neck or when the examination is focused on stenosis diagnostic and if the size of the aneurysm is small.

In diagnosing and characterizing the aneurysms, DSA is still the gold standard imaging method. But, since DSA is an invasive method, persistant neurological complications can develop and that's why nowadays it is mostly used just for endovascular treatment. DSA most often provides the diagnosis of the lesion, specifies the localization, and detects any associated lesion, stenosis, or wall irregularities inducing a carotid dysplasia. The disadvantage of DSA is showing only the patent lumen and the risk of complications associated with invasive catheterization. Therefore, recently noninvasive or minimally invasive methods, such as CT angiography and MR angiography are more popular to detect and demonstrate the aneurysms. 
Contrast enhanced CT scanning with three-dimensional reconstructions allows analysis of the aneurysm and assesses the possible existence of a false lumen channel, representing the existence of a previous dysplastic or traumatic dissection. Analysis of the slices at the osseous window allows the assessment of the distance between the upper limit of the aneurysm and the temporal bone. Currently, contrast enhanced CT scanning with reconstruction is the most sophisticated examination available and gives the most information. CT angiography (CTA) has advantages such as easy and rapid applicability, being a minimally invasive method, having no manifest complication besides contrast medium allergy, capability of rotating the images $360^{\circ}$ [4]. The most important advantage of CT angiography is its capability of evaluating images on preferred planes and angles on the screen. Therefore, superimposed vessel images in DSA making it hard to evaluate are easily evaluated with CT angiography. Moreover, capability of rotating the CT angiographic images on preferred planes and angles helps the surgeon or interventional radiologist in orientation to approach the aneurysm and in the treatment planning (Figure 1). Contrary, $\mathrm{CT}$ angiography has some limitations. Differentiation of small aneurysms from neighboring bones is not possible each time and CT angiography is not capable of showing the collateral circulation as seen in DSA [5]. Both arteries and veins are visible in CT angiography and sometimes it is not easy to differentiate either of them. CT angiography has also limitations in the postoperative management of aneuryms, especially in patients receiving coil embolisation because of coil artefacts.
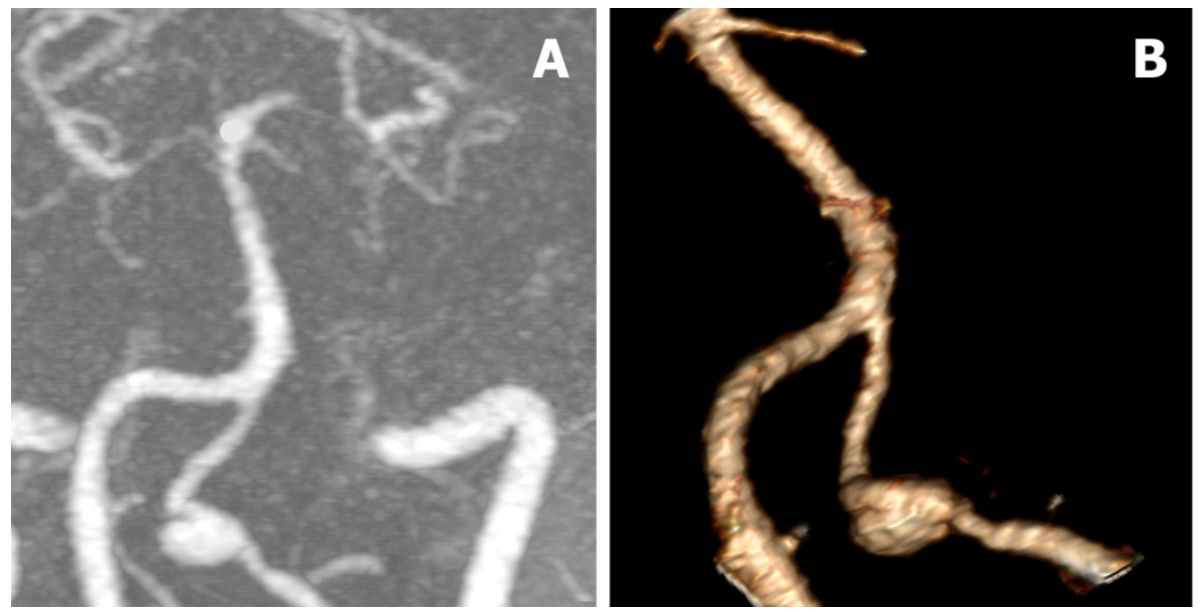

Figure 1. Maximum intensity projection (A) and virtual rendering technique (B) reconstructions of aneurysm of left intradural part of vertebral artery.

Magnetic resonance angiography (MRA) is a non-invasive method that can visualize vascular structures without a need for contrast medium injection or radiation. MRA can manifest the thrombosed portions of aneurysms, residual lumina and flow characteristics. MRA is particularly useful in suspicion of carotid artery dissection due to its characteristic of detecting the old blood in dissected area. MRA and CT angiography mainly replaced the 
conventional angiography. Promiment factors that emphasize superiority of MRA to arteriography are that it excludes the risk of stroke associated with angiography and also possible access site complications and it gives information about the surrounding tissues. MRA also provides reconstruction and rotation of images of intracranial circulation and evaluation of collateral circulation better than angiography.

\subsection{Endovascular treatment}

The current treatment options include surgical treatment and endovascular treatment, but these are not without significant problems [6]. For instance, a randomised, multicentre trial compared the safety and efficacy of endovascular coiling with standard neurosurgical clipping for intracranial aneurysms found that the outcome in terms of survival free of disability at 1 year is significantly better with endovascular coiling [7]. In addition, neurosurgery is associated with significantly longer length of stay and significantly higher total hospital charges [8]. Surgical treatment of extracranial internal carotid artery aneurysms located near skull base is technically challenging with high morbidity and mortality rates. In addition, surgical approach often requires an extended cervicotomy, mandibular subluxation, resection of the styloid process, and sometimes a transection of the external auditory canal with resection of the mastoid and vaginal process of the styloid bone to expose the first vertical intrapetrous segment of the ICA and risk of cranial nerve injury. Over the past decades, with advances in technologies, endovascular therapy is becoming the first-line treatment in the treatment of internal carotid and vertebral artery aneurysms and offers a minimally invasive alternative to open surgery.

Endovascular treatment options includes covered stent placement, flow diverting device (FDD) placement, parent vessel sacrifice with detachable balloons and coils, coil embolisation of the aneurysm with or without a stent placement. Endovascular techniques are usually performed via a femoral access route with placement of either covered stent, FDD or stent extending from the normal artery site to the distal vessel beyond the aneurysm. Despite the trend toward endovascular treatment the rate of recurrence and complications can be high.

This article describes current possibilities in endovascular treatment of vertebral and internal carotid artery aneurysms, with special focus on covered stents with our experience and description of used techniques in the treatment of internal carotid and vertebral artery aneurysms.

\section{Body}

\subsection{CASE 1}

A 52-year-old female with a pulsatile palpable mass in the left retromandibular space was referred to our hospital. Computed tomography angiography revealed a giant false aneurysm of the left cervical segment of the internal carotid artery (ICA) that was probably due to arterial injury caused by an elongated Styloid process. CTA revealed significant 
elongation and tortuosity of the left and right proximal ICA and a large supra-ophthalmic aneurysm of the right ICA (Figure 2).
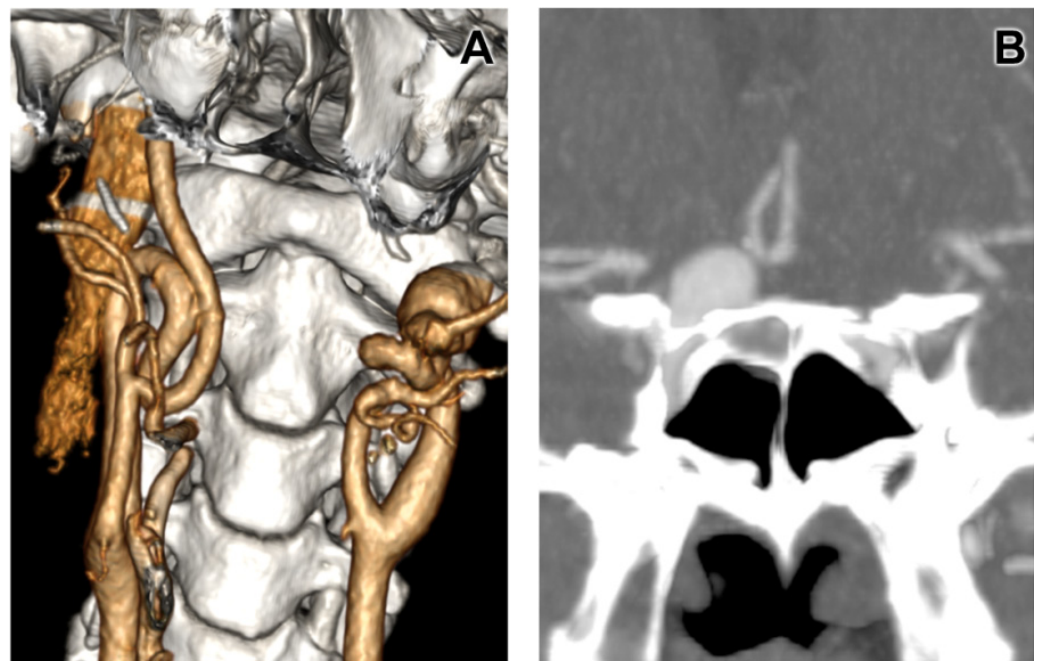

Figure 2. CTA finding of a styloid process causing a false aneurysm of the left ICA, elongation of both proximal parts of the ICA and a large aneurysm of the supra-ophthalmic part of the right ICA $(A, B)$.

A four-step, multidisciplinary therapeutic plan combining surgical and endovascular modalities was selected: (i) resection and straightening of proximal tortuosity of the right ICA; (ii) endovascular coiling of intracranial aneurysms (Figure 3); (iii) resection and straightening of the proximal left ICA; and (iv) endovascular treatment of the false aneurysms in the left retromandibular space using covered stent.
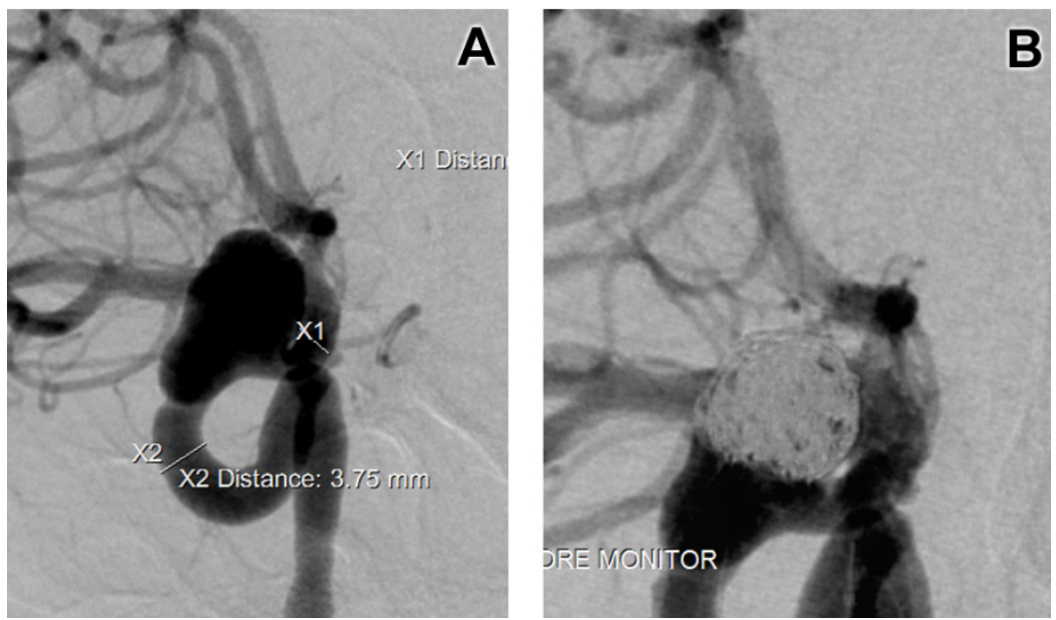

Figure 3. DSA before coiling of the aneurysm of the supra-ophthalmic part of the right ICA (A) and after endovascular treatment (B). 
Before implantation of the pericardium civered stent (PCS), the patient was given $100 \mathrm{mg}$ aspirin and $300 \mathrm{mg}$ clopidogrel. Right femoral access was used and digital subtraction angiography (DSA) of the left carotid artery confirmed the findings of the CTA (Figure 4A, 4B). A 6-F guiding catheter (Guider Softip ${ }^{\mathrm{TM}}$ XF, Boston Scientific Corp., Fremont, CA, USA) was advanced in the distal part of the common carotid artery (CCA). A 0.014 guidewire (Synchro, Boston Scientific Corp., Fremont, CA, USA) was then passed distal to the neck of the aneurysm. The length of the aneurysm neck necessitated two PCS, and resulted in complete exclusion of the aneurysm demonstrated on post-procedure angiography (Figure 4C).
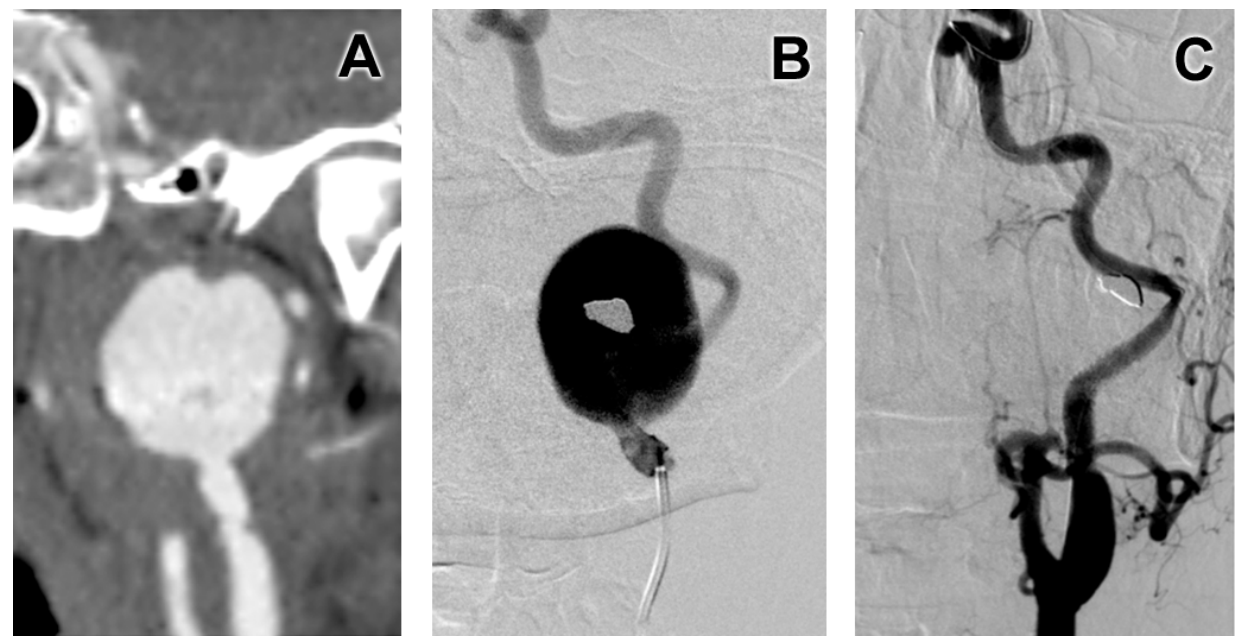

Figure 4. CTA (A) and DSA (B) of a giant false aneurysm of the cervical segment of the left ICA and post-procedure angiography after placement of a covered stent $(\mathrm{C})$.

\subsection{CASE 2}

A 44-year-old female was referred to our hospital after suffering a subarachnoid hemorrhage. Coiling of a small aneurysm of the communicating segment of the left ICA had been done. A giant $(20 \times 18 \mathrm{~mm})$, large-neck aneurysm was discovered on CTA at the intradural fourth segment of the left vertebral artery (VA) proximal to the posterior inferior cerebellar artery (PICA). Endovascular treatment was considered to be first-line treatment for this VA aneurysm. The patient received $100 \mathrm{mg}$ aspirin and $75 \mathrm{mg}$ clopidogrel for 3 days before the procedure. Right femoral access was used and a 6-F guiding catheter (Neuron, Penumbra Inc, San Leandro, California, USA) advanced to the V3 segment of the left VA. DSA showed a giant, large-neck aneurysm of the V4 segment of the VA (Figure 5A). After passage of a 0.014 guidewire (Synchro, Boston Scientific Corp., Fremont, CA, USA) distal to the aneurysm neck, a $4 \times 27 \mathrm{~mm}$ PCS was deployed and inflated to $12 \mathrm{~atm}$. The aneurysm was completely excluded and this was demonstrated at control angiography (Figure 5B). Follow-up CTA at 3 months demonstrated complete exclusion and shrinkage of the aneurysm to $18 \times 16 \mathrm{~mm}$ (Figure 6 ). 

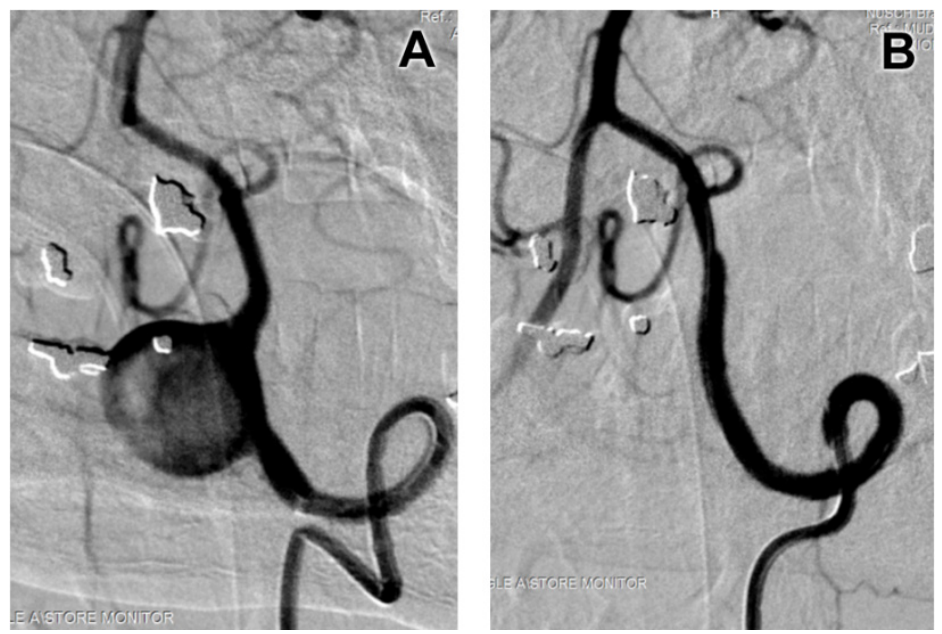

Figure 5. Preoperative DSA of a giant aneurysm of the V4 segment of the left VA (A) and DSA after placement of a covered stent with no filling of the aneurysm (B).

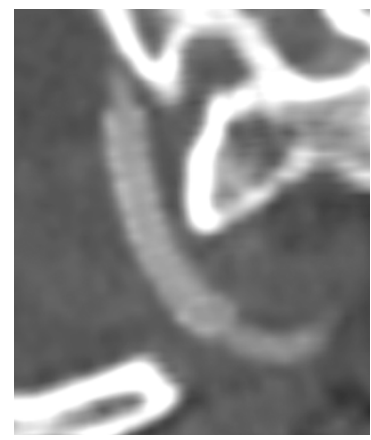

Figure 6. Three-month follow-up CTA showing aneurysm exclusion, a patent covered stent with no intimal hyperplasia, and aneurysm shrinkage.

\subsection{CASE 3}

An 85-year-old female was admitted to our hospital for endovascular therapy of a symptomatic large-neck aneurysm of the cervical segment of the ICA subsequent to a stroke in the left middle cerebral artery (MCA). The patient was pre-medicated with $100 \mathrm{mg}$ aspirin and $75 \mathrm{mg}$ clopidogrel for 3 days before the procedure. Endovascular treatment was undertaken after gaining access via the femoral artery and placement of a 6-F guiding sheath (Guider Softip ${ }^{\mathrm{TM}}$ XF, Boston Scientific Corp., Fremont, CA, USA) in the CCA. DSA confirmed the CTA findings of an aneurysm of the cervical segment of the ICA (Figure 7A, 7B). A 0.014 guidewire (Synchro, Boston Scientific Corp., Fremont, CA, USA) was passed distal to the aneurysm and a PCS $(4 \times 27 \mathrm{~mm})$ advanced over the wire and placed in the optimal position. The balloon was slowly inflated to $10 \mathrm{~atm}$ and the PCS successfully deployed. Control DSA confirmed complete exclusion of the aneurysm with preservation of ICA patency (Figure 7C). 

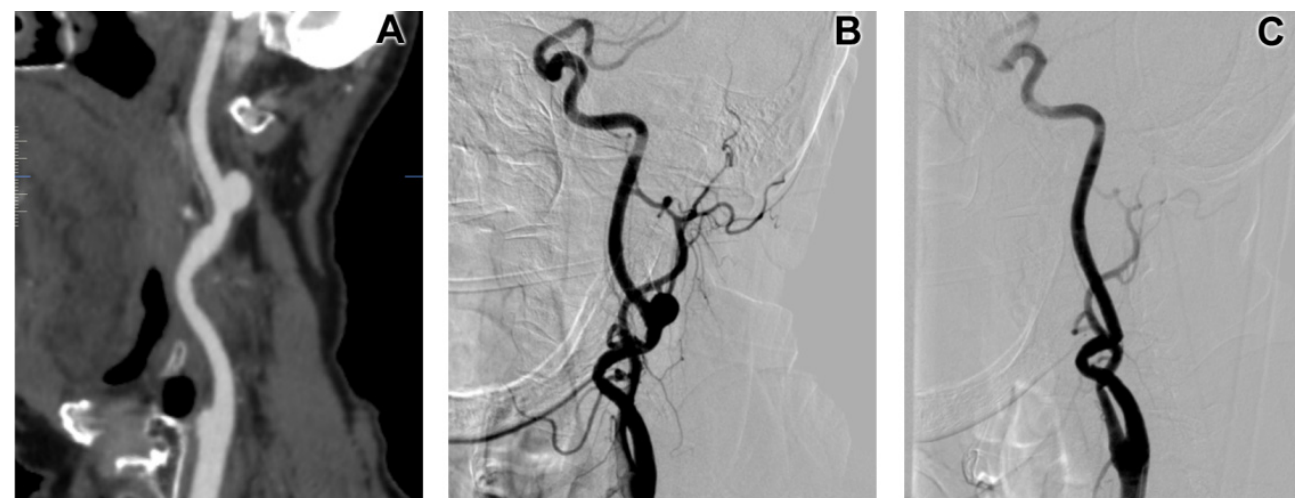

Figure 7. CTA (A) and DSA (B) before endovascular treatment of an aneurysm of the left cervical segment of the ICA and final angiogram after implantation of a covered stent $(\mathrm{C})$.

\subsection{CASE 4}

An 55-year-old male was admitted to our centre for endovascular treatment of a symptomatic dissecting aneurysm of the cervical segment of the ICA subsequent to a stroke. The patient was pre-medicated with $100 \mathrm{mg}$ aspirin and $75 \mathrm{mg}$ clopidogrel for 3 days before the endovascular procedure. Endovascular treatment was performed after gaining access via the right femoral artery and placement of a 6-F guiding sheath (Guider Softip ${ }^{\mathrm{TM}} \mathrm{XF}$, Boston Scientific Corp., Fremont, CA, USA) in the CCA. DSA confirmed the CTA findings of an aneurysm of the cervical segment of the ICA (Figure 8A). A 0.014 guidewire (Synchro, Boston Scientific Corp., Fremont, CA, USA) was passed distal to the aneurysm and a 5x26mm Jostent Graftmaster (Abbott Vascular, Abbott Park, Ill, USA) advanced over the wire and successfully deployed after balloon inflation. Control DSA confirmed exclusion of the aneurysm with patent ICA (Figure 8B).
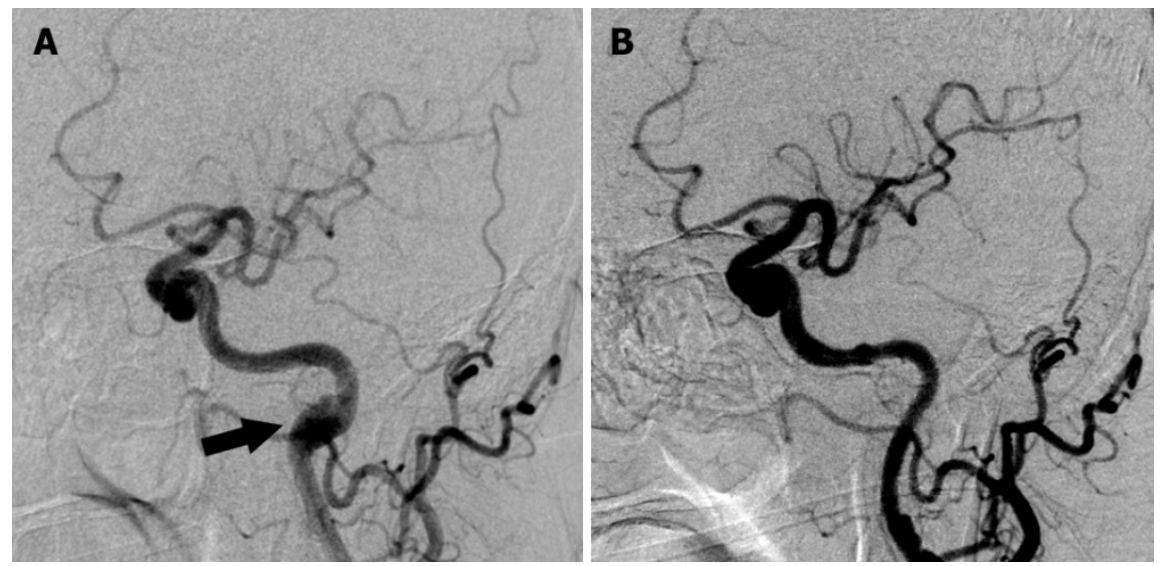

Figure 8. DSA before (A) endovascular treatment of an aneurysm of the left cervical segment of the ICA and angiogram after endovascular treatment with implantation of a covered stent (B). 


\subsection{CASE 5}

A 44-year-old female was referred to our hospital for treatment of an asymptomatic fusiform aneurysm at the intradural fourth segment of the right vertebral artery. Endovascular treatment was considered to be first-line treatment for this VA aneurysm. The patient received $100 \mathrm{mg}$ aspirin and $75 \mathrm{mg}$ clopidogrel for 3 days before the procedure. Left femoral access was used and a 6-F guiding catheter (Neuron, Penumbra Inc, San Leandro, California, USA) advanced to the V3 segment of the right VA. DSA confirmed fusiform aneurysm of the V4 segment of the VA (Figure 9A). After passage of a 0.014 guidewire (Synchro, Boston Scientific Corp., Fremont, CA, USA) distal to the aneurysm neck, a flow diverting device Silk ((Balt, Montmorency, France) was deployed. The aneurysm was excluded and this was demonstrated at control angiography with no filling of the aneurysm (Figure 9B).

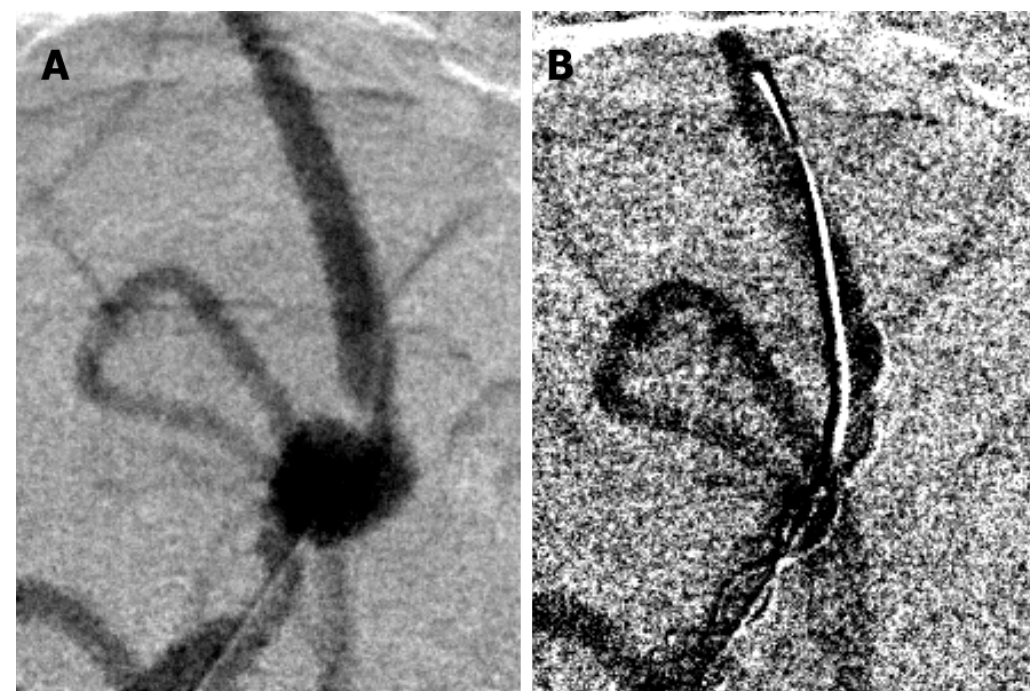

Figure 9. DSA before (A) endovascular therapy of an aneurysm of the intradural segment of right vertebral artery and angiogram after implantation of flow diverting device (B).

\section{Discussion}

Different endovascular techniques have been used in the treatment of vertebral and internal carotid artery aneurysms. These include covered stent placement, flow diverting device (FDD) placement, parent vessel occlusion with detachable balloons or coils, coil embolisation of the aneurysm with or without a stent placement.

\subsection{Covered stents}

In the past decade, covered stent placement has been proved to be effective for managing arteriovenous fistulas, aneurysms, and aortic dissections. Covered stents consist of a 
synthetic material that either covers or is attached to a metallic stent to create a graft endoprosthesis. There are two different types of stent-deployment system - balloonexpandable or self-expanding. Balloon-expandable system represent Jostent Graftmaster (Abbott Laboratories, Abbott Park, Ill) and iCast (Atrium Medical, Hudson, NH), selfexpanding covered stents are Fluency stent (Bard Peripheral Vascular, Tempe, Ariz), GORE VIABAHN (W.L. Gore \& Assoc, Newark, Del), Wallgraft (Boston Scientific, MA) and Willis covered stent (MicroPort, Shanghai, China). Compared with aneurysm coil embolization, a covered stent has the following advantages: (1) a relatively simple and rapid performance; (2) a low risk of procedural-related rupture or rebleeding; (3) no coil herniation, delayed migration, and coil loop protrusion; (4) disappearance or reduction of mass effects in large or giant aneurysms; and (e) no aneurysm recanalization and recurrence.

The Jostent graft is a composite stent with an ultrathin layer of expandable PTFE sandwiched between two stainless steel stents. It is balloon-expandable, covered-tube stent with radial strength. Jostent has been used for aneurysms up to the ophthalmic artery or up to the vertebrobasilar junction [9,10]. The Graftmaster has also been used for carotidocavernous fistulas and dissections [11]. Bergeron et al. recently published a series of six patients with EICA in which stent-grafts were used. They had no adverse event perioperatively and the long follow-up revealed patency of all grafts without sign of in-stent stenosis [12]. Chan et al. also reported two cases of successful endovascular treatment of distal internal carotid aneurysms using Jostent, with both patients remaining symptom-free at 1 year and no signs of graft restenosis [13]. Th disadvantage of Jostent is, that it might cause intimal damage at the stent edges as a result of irritation from vessel motion or anatomic distortion and also requires a relatively straight delivery path and is not well suited for use in very tortuous vessels.

The Willis covered stent is specifically designed for use in the intracranial vasculature and consists of 3 parts: a bare stent, an expandable polytetrafluoroethylene (ePTFE) membrane, and a balloon catheter. The bare stent was constructed from a strand of cobalt chromium super alloy wire, which was $0.06 \mathrm{~mm}$ in diameter. The ePTFE membrane, which was in a tubular configuration with a thickness of 30 to $50 \mu \mathrm{m}$ is glued along the length of the stent struts with use of organic agglomerate. To facilitate the membrane gluing along the stent, the diameter of tubular membrane is generally $0.05 \mathrm{~mm}$, which is wider than that of the inflated stent. To prevent the balloon from scaling the inner wall of the stent on withdrawal, the balloon is made into 5 valvae, instead of the commonly used 3 valvae. The whole body of the stent is radiopaque under fluoroscopy to facilitate precise placement of the stent. The stent can be manufactured in any diameter from 3 to $5 \mathrm{~mm}$ and in any length from 7 to 15 $\mathrm{mm}$. The stent is mounted on a deflated balloon catheter with an outside diameter of 3.8F. $\mathrm{Li}$ at. al. performed successfull endovascular treatment of pseudoaneurysms of cranial internal carotid artery in 8 patients without procedural-related complications, and all of the stents were easily navigated to the targeted lesions. Complete resolution of the pseudoaneurysm was observed in 6 patients immediately after the procedure, and a minimal endoleak into the aneurysm persisted in 2 patients. No morbidity or mortality and no technical adverse event occurred. A follow-up angiogram confirmed complete 
reconstruction of the internal carotid artery, with no recurrent aneurysmal filling and no occurrence of stenosis in the area of the stent [14]. The efficacy of the Willis covered stent in the treatment of traumatic pseudoaneurysms of the internal carotid artery was evaluated in 13 patients with 14 delayed pseudoaneurysms with succesfull covered stent placement in all 14 pseudoaneurysms. The initial angiographic results showed complete exclusion in 9 patients with 10 aneurysms and incomplete exclusion in 4 patients. The angiographic mean follow-up 15 months findings exhibited a complete exclusion in 12 patients with 13 aneurysms and an incomplete exclusion in 1 patient and maintained patency of the ICA in all patients with no procedure-related complications or deaths occurred during follow-up [15]. In another study, the navigation and deployment of the Willis covered stents were successful in $97.6 \%$ (41 of 42 ) of the patients with most of the aneurysms located at the C5 through C7 segment. Although some complications occurred, the 93.5\% (29 of 31 aneurysms) final complete occlusion rate with no recanalization during follow-up addressed the effectiveness of the Willis covered stent for managing DICA aneurysms. In addition, endoleak occurred in $21.9 \%$ (7 of 32) of the patients owing to the tortuous segment of C5 through $\mathrm{C} 7$, but it could not been eliminated by means of postprocedural dilation and additional stent implantation [16].

The Wallgraft consists of a PET (Dacron; E.I. duPont de Nemours and Co., Wilmington, DE) covered self-expanding cobalt super alloy stent. Wallgraft was utilized in 4 cases of inernal carotid artery trauma or disease leading to contained rupture or pseudoaneurysm formation. During a mean 16-month follow-up (range 6-24), duplex ultrasound and CT scanning found no evidence of restenosis, occlusion, or persistent perfusion of the pseudoaneurysm, which was noted to decrease in all cases [17]. Wallgraft was also used in the treatment of tandem aneurysms of the extracranial internal carotid artery near the skull base with successfully exclusion with deployment of a single Wallgraft across both lesions with no complications encountered. At 2-year follow-up, the patient is doing well, without any sign of aneurysm reperfusion [18]. The Wallgraft has several disadvantages. First, PET is immunogenic which animal studies suggest, increases the rate of vessel thrombosis [19]. Second, the Wallgraft delivery system requires a 9-French arterial sheath in the carotid and vertebral arteries, which may increase the risk of pseudoaneurysms or groin hematomas at the femoral puncture site. Finally, the PET covering is initially porous until a clot forms to seal the fabric.

Viabahn endograft is a PTFE covered nitinol stent with extreme flexibility and conformability to vessel configuration. Covered stents have also shown better closure and shorter procedure time in clinical investigations [20]. Current published evidence of the use of covered stent is limited to stents covered with polytetrafluoroethylene (PTFE) [21]. Synthetic materials are used in biological settings with limited success. Studies have shown that polyester covered stent graft with $50 \%$ re-stenosis and e-PTFE covered stent with $24 \%$ re-stenosis in a sheep iliac model [22]. It has also been shown that PTFE retards endothelization and that Dacron is prone to infection, due to adherence to and survival of bacteria on its rough surface [23]. Baldi et al. in their three cases had no periprocedural complications and all three PTFE Viabahn endografts were patent at 9 month follow-up without evidence of intimal hyperplasia [24]. 
A novel CE marked coronary stent covered with pericardium (Aneugraft: ITGI Medical limited, Or Akiva, Israel) is available, which so far shows promising clinical results. The pericardium covered stent (PCS) is a percutaneous implantable device consisting of a 316L stainless steel stent covered by a $100 \mu$ thick equine pericardium cylinder which makes this device flexible and trackable. It is available in diameters of $2.5 \mathrm{~mm}, 3.0 \mathrm{~mm}, 3.5 \mathrm{~mm}$, and $4.0 \mathrm{~mm}$, and lengths of $13,18,23$ and $27 \mathrm{~mm}$. The stent is mounted on a balloon catheter. Gluteraldehyde treated pericardium has been widely used for many years due to its desirable features such as low immunogenicity and durability [25-27]. It has been shown that there is significantly less inflammatory cytokine, significantly less antibody response and inflammatory response compared to un-crosslinked decellularized pericardium [28]. It is now recognized that mammalian extracellular matrix represents an excellent scaffold material suitable for many therapeutic applications [29]. In Neurosurgery, serous sheets are used as dural substitute. An investigation involving 200 patients undergoing a surgical procedure with the application of horse pericardium as a dural prosthesis found that they are free from antigenic effects and do not produce any toxic catabolites [30]. The pericardium proved to be resistant to surgical suture, impermeable to cerebrospinal fluid, transparent and does not cause any clinical evidence or radiological artifacts. Pericardium has also shown decreased intraoperative suture line bleeding compared to Dacron [31]. The PCS has shown to be safe and effective in 2 registries [32], and there is also published evidence attesting to this in other indications [33-36].

There are several disadvantages regarding the use of covered stents in the cranial and extracranial vasculature. First, more clinical trials are required to determine the long-term outcomes. Second, the covered stents may not be flexible and conformable enough to navigate entirely through the extremely tortuous ICA and to fully conform to the configuration of the tortuous targeted arteries. Third, the possibility of a closure of the side branches stemming from the covered segment of the artery might occur after the stent placement. Therefore, balloon occlusion tests and angiography examinations from multiple projections should be routinely performed to avoid coverage of the side branches. In addition, in-stent restenosis might occur in patients who are not following the regular anticoagulation medication regimen after stent placement.

\subsection{FDD}

Flow diversion is a new approach to the endovascular treatment of intracranial aneurysms which uses a high density mesh stent to induce sac thrombosis. These devices have been designed for the treatment of complex shaped and large size aneurysms. Flow diversion aims to cure aneurysms by endovascular reconstruction of the parent vessel, without even performing endosaccular embolisation. The primary intent of a flow diversion device (as opposed to a stent) is to optimally alter the flow exchange between the parent artery and the aneurysm so as to promote complete thrombosis of the sac as rapidly as possible while eliciting minimal neointimal hyperplasia. The principal goal of the flow divertor is placement in the parent artery in order to reconstruct the vessel wall [37]. The concept of flow diversion appears promising in challenging lesions, including fusiform and/or giant 
aneurysms. However, this stent presents major limitations: (a) the aneurysm occlusion process is unpredictable; (b) an associated complication rate much higher than those previously reported with conventional treatments (coiling, balloon- or stent-assisted coiling, parent artery occlusion, clipping); and (c) a high rate of significant parent artery stenosis.

In contrast to an ideal stent, an ideal flow diversion device has low porosity and high pore density values optimized to promote intraaneurysmal thrombosis while maintaining patency of the parent vessel and side branches. Moreover, lower radial forces are required of this device as compared to a stent, which facilitates the optimization of other device characteristics such as longitudinal flexibility, trackability, and conformability. Recognition of the potential for aneurysm treatment by flow diversion is evidenced by the recent development of these devices by various groups.The major complications with flow divertors have been found to be perforator artery stroke, aneurysm re-rupture, and in-stent stenosis and thrombosis [38,39].

The Pipeline stent (EV3, Irvine, Calif) is the first released flow-diverter stent and it has been evaluated in some series $[40,41]$. These authors showed that the Pipeline stent represents a safe, durable, and curative treatment of selected wide-necked, large, and giant aneurysms. The Pipeline stent has been used for the treatment of two male patients transferred after acute SAH and dissecting aneurysm on the V4 segment of the dominant vertebral artery with 3 Pipeline stents deployed in each vertebral artery. One dissecting aneurysm was excluded immediately after 3 stents and one patient had complete exclusion demonstrated at the 48 hour control. No morbidity directly related to the procedure was observed and no recanalization and no re-bleeding occurred during the 3 months follow-up [42]. In the recent publication, Yeung et al. demonstrated favourable long-term clinical and angiographic outcomes of FDD use and the ability to maintain parent artery and side branch patency for the endovascular treatment of unruptured dissecting intracranial vertebral aneurysms. In their series, total of 4 aneurysms were successfully obliterated by using flowdiverting devices alone, two devices were deployed in a telescoping fashion in each of 2 aneurysms, whereas only 1 device was inserted in each of the other 2 aneurysms with no periprocedural complications. No patient showed any angiographic evidence of recurrence, in-stent thrombosis, or side-branch occlusion in angiographic reassessment at a mean of 22 months after treatment (range 18-24 months) [43].

The other available flow-diverter device is the Silk stent (Balt, Montmorency, France) and little information is available concerning its use [44-46]. By the use of telescopic catheters, the Silk stent may be placed in most patients. Silk opening and wall apposition frequently require pushing back the delivery catheter. This is particularly mandatory within curved vessel such as the ICA siphon. Because of its low radial force, the Silk stent must be placed with great caution if the vessel shows a stenotic portion because vessel occlusion may occur. Moreover, careful size and length selection is mandatory because stent shortening and migration may happen. For all these reasons, Silk stent placement is more difficult than nonflow-diverter self-expandable stent. 


\subsection{Parent vessel occlusion}

One of the treatment options available for patients with internal carotid or vertebral artery aneurysms is parent vessel occlusion, either surgical or endovascular. The goal of parent vessel occlusion for the treatment of fusiform aneurysms is intra-aneurysmal thrombosis and involution of the aneurysm. Endovascular occlusion can be achieved with detachable balloons or coils or with a combination of the two. Studies reporting patient outcomes after parent vessel occlusion for treatment of fusiform aneurysms of the vertebrobasilar circulation have been limited. A few series have reported the results of parent vessel occlusion in the posterior circulation, although not exclusively for intracranial fusiform aneurysms. In one study the long-term outcomes for 21 patients with unclippable posterior circulation aneurysms treated with either unilateral or bilateral parent vessel occlusion of the vertebral artery, with a mean follow-up of 2 years (range, 6 months to 6 years) were examined [47]. Six of the patients had fusiform aneurysms, and the remaining 5 had aneurysms that were of saccular morphology. All occlusions in this series were performed by using latex balloons. Thirteen $(61.9 \%)$ of 21 patients achieved good outcomes, including angiographic cure and clinical improvement. Twenty-eight and six-tenths percent of the patients had partial thrombosis of their aneurysm. One death and one treatment failure occurred.

Occlusion of the internal carotid artery may lead to severe cerebrovascular events and therefore a balloon occlusion test should be performed in advance; if a temporary occlusion test is successful, trapping or parent artery occlusion is an option. However, it has been shown that $5-22 \%$ of patients passing the balloon occlusion test develop ischemic complications, including cerebral infarct, while some reports have revealed cerebral aneurysm formation after permanent carotid occlusion [48,49]. The placement of detachable balloons in the ICA above and below the false aneurysm can completely eliminate blood flow. Disadvantages with this endovascular approach include the possibility of embolic cerebrovascular accidents. If the patient cannot tolerate the occlusion test, an extracranial-tointracranial bypass should be contemplated.

\subsection{Coil embolisation and stent placement}

Another treatment option in the management of aneurysms represents stent placement with or without coil embolisation and coil embolisation without stent placement. Findings of experimental studies have shown that a metallic stent, bridging the aneurysmal neck, may alter the flow pattern within the aneurysm, promoting thrombus formation and aneurysmal occlusion [50,51]. Although immediate aneurysmal occlusion can be seen after single stent placement for treatment of extracranial pseudoaneurysms, in some cases, 3-6 months or longer may pass before occlusion occurs. To achieve faster complete aneurysmal occlusion, the combination of stents and detachable coils has been suggested for extracranial, as well as intracranial aneurysms $[2,52,53]$ and the combination is currently considered an alternative to single stent placement or other techniques such as the remodeling technique or parent vessel occlusion. Lanzino et al [52] reported 10 cases managed with stent-supported coil 
embolization; they achieved aneurysmal occlusion of more than $90 \%$ in eight patients. In four of these patients, treated with stent placement only, no evidence of intraaneurysmal thrombosis was found either immediately or during follow-up studies performed 48 hours (two patients), 4 days (one patient), and 3 months (one patient) after treatment. Phatouros et al [2] reported a series of seven patients with fusiform aneurysms, wide-neck aneurysms, or pseudoaneurysms who underwent stent-supported coil embolization; technical success was achieved in six. In one patient, a coil became entangled with the stent, resulting in partial coil delivery into the parent artery with no neurologic sequelae.

However, certain limitations, may be encountered when stents are used in conjunction with coils. In some cases, a microcatheter can be navigated through the stent interstices only with difficulty. Packing of the aneurysm sac can be inaccurate because of the density of platinum detachable coils. Multiple projections with big amount of used contrast medium may become necessary, particularly with fusiform aneurysms, and in some cases, complete packing of the aneurysm cannot be achieved. Coil loops may become entangled with the stent struts and unravel during attempts to retrieve them, leading to the risk of moving the stent or leaving coils within the parent artery. A report by Phatouros et al [2] shows the successful use of stent-supported coil embolization in the treatment of fusiform and wide neck aneurysms. The stent mesh allows for attenuated packing of the aneurysm with less concern for herniation of coils into the parent artery. Phatouros et al reported technical success in six of the seven patients treated, with $0 \% 30$-day periprocedural morbidity and mortality. After a mean follow-up of 14.5 months, all the patients treated with stentsupported coil embolization were at their neurologic baseline or had improved. The authors acknowledged the current limitations of this therapy, including the concern for occluding small but important perforators with the struts of the stent. Kurata et al. published their findings in a series of 24 ruptured dissecting vertebral artery aneurysms. Endosaccular embolisation was performed within 4 days of onset of symptoms with no experienced complications with coil embolisation. Radiologic investigation showed complete occlusion of the dissection and patency of the unaffected artery at a mean follow-up of 9 months [54].

The use of double stent placement for complete exclusion of wide-neck aneurysms has been reported in only a single case to date [55], however double stent placement may be a relatively simple technique to more effectively change intraaneurysmal flow and achieve subsequent thrombosis. The influence of stent porosity on changing the local hemodynamics between the aneurysm and the parent vessel was shown in the experimental study [56].

\section{Conclusion}

In conclusion, endovascular treatment of vertebral and carotid artery aneurysms with covered stents is very promising, safe and feasible treatment option. So called flow diverting devices despite their "slow mode" of action, but according to their special features (as is high flexibility and very low profile), seems to be very effective tool in endovascular treatment of carotid and vertebral artery aneurysms. On the other hand, covered stents with for example a novel pericardium covered stent, allow complete occlusion of the aneurysm, 
fistula or dissection in one action. This approach in the treatment of aneurysms seems to be very promising. Therapeutic decision making in the treatment of vertebral and carotid artery aneurysms must balance endovascular or surgical morbidity and mortality rates with the risk of hemorrhage and other considerations on an individual basis. Evolving technologies move towards increased covered stents flexibility with pushing down their profiles. This evolution followed with future studies of these advanced endovascular approaches will probably increase the role of covered stents in the field of endovascular treatment of vertebral and carotid artery aneurysms in the future. More detailed clinical studies will need to be conducted to confirm the overall performance and long-term effect of covered stents in the treatment of internal carotid and vertebral artery aneurysms.

\section{Author details}

Ivan Vulev and Andrej Klepanec

Department of Diagnostic and Interventional Radiology,

National Institute of Cardiovascular Diseases Bratislava, Slovakia

\section{References}

[1] Vega C, Kwoon JV, Lavine SD. Intracranial aneurysms: current evidence and clinical practice. American Family Physician 2002;66(4): 601-608.

[2] Phatouros CC, Sasaki TY, Higashida RT, Malek AM, Meyers PM, Dowd CF, Halbach VV. Stent-supported coil embolization: the treatment of fusiform and wide-neck aneurysms and pseudoaneurysms. Neurosurgery 2000;47: 107-115.

[3] Nichols DA, Brown RD, Thielen KR, Meyer FB, Atkinson JL, Piepgras DG. Endovascular treatment of ruptured posterior circulation aneurysms using electrolytically detachable coils. Journal of Neurosurgery 1997;87: 374-380.

[4] Matsumoto M, Sato M, Nakano M, Endo Y, Watanabe Y, Sasaki T, Suzuki K, Kodama $\mathrm{N}$. Three-dimensional computerized tomography angiography-guided surgery of acutely ruptured cerebral aneurysms. Journal of Neurosurgery 2001;94: 718-727.

[5] Villablanca JP, Martin N, Jahan R, Gobin YP, Frazee, Duckwiler G, Bentson J, Hardart M, Coiteiro D, Sayre J, Vinuela F. Volume rendered helical computerized tomography angiography in the detection and characterisation of intracranial aneurysms. Journal of Neurosurgery 2000;93: 254-264.

[6] Rinkel GJ. Natural history, epidemiology and screening of unruptured intracranial aneurysms. Journal of Neuroradiology 2008;35(2): 99-103.

[7] Molyneux A, Kerr R, Stratton I, Sandercock P, Clarke M, Shrimpton J, Holman R; International Subarachnoid Aneurysm Trial (ISAT) Collaborative Group. International Subarachnoid Aneurysm Trial (ISAT) of neurosurgical clipping versus endovascular coiling in 2143 patients with ruptured intracranial aneurysms: a randomised trial. Lancet 2002;60: 1267-1274.

[8] Hoh BL, Chi YY, Lawson MF, Mocco J, Barker FG 2nd. Length of stay and total hospital charges of clipping versus coiling for ruptured and unruptured adult cerebral 
aneurysms in the Nationwide Inpatient Sample database 2002 to 2006. Stroke 2010;41(2): 337-342.

[9] Saatci I, Cekirge SH, Ozturk MH, Arat A, Ergungor F, Sekerci Z, Senveli E, Er U, Turkoglu S, Ozcan OE, Ozgen T. Treatment of Internal Carotid Artery Aneurysms with a Covered Stent: Experience in 24 Patients with Mid-Term Follow-up Results. American Journal of Neuroradiology 2004;25: 1742-1749.

[10] Greenberg E, Katz, JM, Janardhan, V, Riina, H, Gobin, YP. Treatment of a giant vertebrobasilar artery aneurysm using stent grafts. Journal of Neurosurgery 2007;107: 165-168.

[11] Gomez F, Escobar W, Gomez AM, Gomez JF, Anaya CA. Treatment of Carotid Cavernous Fistulas Using Covered Stents: Midterm Results in Seven Patients. American Journal of Neuroradiogy 2007;28: 1762-1768.

[12] Bergeron P, Khanoyan P, Meunier J-P, Graziani JN, Gay J. Long term results of endovascular exclusion of extracranial internal carotid artery aneuryms and dissecting aneurysm. Journal of Interventional Cardiology 2004;17: 245-252.

[13] Chan AW, Yadav JS, Krieger D, Abou-Chebl A. Endovascular repair of carotid artery aneurysm with Jostent covered stent: Initial experience and one year result. Catheteterization and Cardiovascular Interventions 2004;63: 15-20.

[14] Li MH, Li YD, Gao BL, Fang C, Luo QY, Cheng YS, Xie ZY, Wang YL, Zhao JG, Li Y, Wang W, Zhang BL, Li M. A new covered stent designed for intracranial vasculature: application in the management of pseudoaneurysms of the cranial internal carotid artery. American Journal of Neuroradiology 2007;28(8): 1579-1585.

[15] Wang, W, Li MH, Li YD, Gu BX, Wang J, Zhang PL, Li M. Treatment of Traumatic Internal Carotid Artery Pseudoaneurysms With the Willis Covered Stent: A Prospective Study. Journal of Trauma-Injury Infection and Critical Care 2011;70(4): 816-822.

[16] Li MH, Li YD, Tan HQ, Luo QY, Cheng YS. Treatment of Distal Internal Carotid Artery Aneurysm with the Willis Covered Stent: A Prospective Pilot Study. Radiology 2009;253: 470-477.

[17] Kubaska SM 3rd, Greenberg RK, Clair D, Barber G, Srivastava SD, Green RM, Waldman DL, Ouriel K. Internal Carotid Artery Pseudoaneurysms: Treatment With the Wallgraft Endoprosthesis. Journal of Endovascular Therapy 2003;10(2): 182-189.

[18] Fischer B, Palkovic S, Wassmann H. Endovascular Management of Tandem Extracranial Internal Carotid Artery Aneurysms With a Covered Stent. Journal of Endovascular Therapy 2004;11: 739-741.

[19] Hussain FM, Kopchok G, Heilbron M, Daskalakis T, Donayre C, White RA. Wallgraft endoprosthesis: Initial canine evaluation. The American Surgeon 1998;64: 1002-1006.

[20] Li MH, Leng B, Li YD, Tan HQ, Wang W, Song DL, Tian YL. Comparative study of covered stent with coil embolization in the treatment of cranial internal carotid artery aneurysm: a nonrandomized prospective trial. European Radiology 2010;20(11): 27322739.

[21] Tan HQ, Li MH, Zhang PL, Li YD, Wang JB, Zhu YQ, Wang W. Reconstructive endovascular treatment of intracranial aneurysms with the Willis covered stent: 
medium-term clinical and angiographic follow-up. Journal of Neurosurgery 2011;114(4): 1014-1020.

[22] Cejna M, Virmani R, Jones R. Biocompatibility and Performance of the Wallstent and Several Covered Stents in a Sheep Iliac Artery Model. Journal of Vascular and Interventional Radiology 2001;12: 351-358.

[23] Shintani H. Modification of Medical Device Surface to attain Anti-Infection Trends. Biomaterials, Artificial Cells, and Artificial Organs 2004;18(1): 1-8.

[24] Baldi S, Rostagno RD, Zander T, Llorens R, Schonholz C, Maynar M. Endovascular Treatment of Extracranial Internal Carotid Aneurysms Using Endografts. Cardiovascular and Interventional Radiology 2008;31: 401-403.

[25] Butany J, Luk A, Leong SW, Leong MM, Singh G, Thangaroopan M, Williams W. A Carpentier-Edwards porcine-valved dacron conduit: at twenty-five years. International Journal of Cardiology 2007;117(1): 13-16.

[26] Jamieson WR, Burr LH, Munro AI, Miyagishima RT. Carpentier-Edwards standard porcine bioprosthesis: a 21-year experience. Annals of Thoracic Surgery 1996;66(6 Suppl): S40-3.

[27] Cribier A, Eltchaninoff, Tron C. Early experience with percutaneous transcatheter implantation of heart valve prosthesis for the treatment of end-stage inoperable patients with calcific aortic stenosis. Journal of the American College of Cardiology. 2004;43: 698-703.

[28] Umashankar PR, Arun T, Kumari TV. Short duration gluteraldehyde cross linking of decellularized bovine pericardium improves biological response. Journal of Biomedical Materials Research Part A 2011;97(3): 311-320.

[29] Badylak SF, Freytes DO, Gilbert TW. Extracellular matrix as a biological scaffold material: Structure and function. Acta Biomaterialia 2009;5(1): 1-13.

[30] Montinaro A, Gianfreda CD, Proto P. Equine pericardium for dural grafts: clinical results in 200 patients. Journal of Neurosurgical Sciences 2007;51(1): 17-19.

[31] Marien BJ, Raffetto JD, Seidman CS, LaMorte WW, Menzoian JO. Bovine pericardium vs dacron for patch angioplasty after carotid endarterectomy: a prospective randomized study. Archives of Surgery 2002;137(7): 785-788.

[32] Colombo A, Almagor Y, Gaspar J, Vonderwalde C. The pericardium covered stent (PCS). EuroIntervention 2009;5(3): 394-399.

[33] Summaria F, Romagnoli E, Preziosi P. Percutaneous antegrade transarterial treatment of iatrogenic radial arteriovenous fistula. Journal of Cardiovascular Medicine (Hagerstown) 2012;13(1): 50-2.

[34] Adlam D, Hutchings D, Channon KM. Optical coherence tomography-guided stenting of a large coronary aneurysm: images at implantation and at 6 months. J. Invasive Cardiol. 2011;23(4): 168-169.

[35] Siregar S, Hoseyni Guyomi S, van Herwerden LA. Covered stents in giant coronary artery aneurysm. European Heart Journal 2010;31(22): 2823.

[36] Ferlini M, Russo F, Marinoni B, Repetto A, Canosi U, Ferrario M, Visconti LO, Bramucci E. Percutaneous coronary aneurysm obliteration using a novel pericardium-covered stent. Journal of the American College of Cardiology 2010;14;56(25): 2139. 
[37] Wong GK, Kwan MC, Ng RY, Yu SC, Poon WS. Flow diverters for treatment of intracranial aneurysms: Current status and ongoing clinical trials. Journal of Clinical Neuroscience 2011; 18(6):737-740.

[38] Walcott BP, Pisapia JM, Nahed BV, Kahle KT, Ogilvy CS. Early experience with flow diverting endoluminal stents for the treatment of intracranial aneurysms. Journal of Clinical Neuroscience 2011;18(7): 891-894.

[39] Byrne JV, Beltechi R, Yarnold JA, Birks J, Kamran M. Early experience in the treatment of intra-cranial aneurysms by endovascular flow diversion: a multicentre prospective study. PLoS One 2010;5(9): e12492.

[40] Fiorella D, Woo HH, Albuquerque FC, Nelson PK. Definitive reconstruction of circumferential, fusiform intracranial aneurysms with the pipeline embolization device. Neurosurgery 2008;62: 1115-1120.

[41] Lylyk P, Miranda C, Ceratto R, Ferrario A, Scrivano E, Luna HR, Berez AL, Tran Q, Nelson PK, Fiorella D. Curative endovascular reconstruction of cerebral aneurysms with the pipeline embolization device: the Buenos Aires experience. Neurosurgery 2009;64: 632-642.

[42] Narata AP, Yilmaz H, Schaller K, Lovblad KO, Pereira VM. Flow Diverter Stent for Ruptured Intracranial Dissecting Aneurysm of Vertebral Artery. Neurosurgery 2011;70(4): 982-989.

[43] Yeung TW, Lai V, Lau HY, Poon WL, Tan CB, Wong YC. Long-term outcome of endovascular reconstruction with the Pipeline embolization device in the management of unruptured dissecting aneurysms of the intracranial vertebral artery. Journal of Neurosurgery 2012;116(4):882-887.

[44] Appelboom G, Kadri K, Hassan F, Leclerc X. Infectious aneurysm of the cavernous carotid artery in a child treated with a new-generation of flow-diverting stent graft: case report. Neurosurgery 2010;66: 623-624.

[45] Turowski B, Macht S, Kulcsár Z, Hänggi D, Stummer W. Early fatal hemorrhage after endovascular cerebral aneurysm treatment with a flow diverter (SILK-Stent): do we need to rethink our concepts? Neuroradiology. 2011;53(1): 37-41.

[46] Lubicz B, Collignon L, Raphaeli G, Pruvo JP, Bruneau M, De Witte O, Leclerc X. FlowDiverter Stent for the Endovascular Treatment of Intracranial Aneurysms A Prospective Study in 29 Patients With 34 Aneurysms. Stroke 2010;41: 2247-2253.

[47] Aymard A, Gobin YP, Hodes J, Bien S, Rüfenacht D, Reizine D, George B, Merland JJ. Endovascular occlusion of vertebral arteries in the treatment of unclippable vertebrobasilar aneurysms. Journal of Neurosurgery 1991;74: 393-398.

[48] Eckert B, Thie A, Carvajal M, Groden C, Zeumer H. Predicting hemodynamic ischemia by transcranial Doppler monitoring during therapeutic balloon occlusion of the internal carotid artery. American Journal of Neuroradiology 1998;19: 577-582.

[49] Larson JJ, Tew JM Jr, Tomsick TA, van Loveren HR. Treatment of aneurysms of the internal carotid artery by intravascular balloon occlusion: Long-term follow-up of 58 patients. Neurosurgery 1995;36: 26-30. 
[50] Geremia G, Haklin M, Brennecke L. Embolization of experimentally created aneurysms with intravascular stent devices. American Journal of Neuroradiology 1994;15: 12231231.

[51] Wakhloo AK, Schellhammer F, de Vries J, Haberstroh J, Schumacher M. Self-expanding and balloon-expandable stents in the treatment of carotid aneurysms: an experimental study in a canine model. American Journal of Neuroradiology 1994;15: 493-502.

[52] Lanzino G, Wakhloo AK, Fessler RD, Hartney ML, Guterman LR, Hopkins LN. Efficacy and current limitations of intravascular stents for intracranial internal carotid, vertebral, and basilar artery aneurysms. Journal of Neurosurgery 1999;91: 538-546.

[53] Lylyk P, Ceratto R, Hurvitz D, Basso A. Treatment of a vertebral dissecting aneurysm with stents and coils: technical case report. Neurosurgery 1998;43: 385-388.

[54] Kurata A, Ohmomo T, Miyasaka Y, Fujii K, Kan S, Kitahara T. Coil embolisation for the treatment of ruptured dissecting vertebral aneurysms. American Journal of Neuroradiology 2001;22: 11-18.

[55] Benndorf G, Lehmann T, Schneider G, Wellnhofer E. Double stenting: technique to accelerate occlusion of a dissecting carotid artery aneurysm. Neuroradiology 1999;41: (suppl) 77.

[56] Lieber BB, Stancampiano AP, Wakhloo AK. Alteration of hemodynamics in aneurysm models by stenting: influence of stent porosity. Annals of Biomedical Engineering 1997;25: 460-469. 